\title{
Short Course Organizer Takes the Long View on ICME Implementation
}

\section{Lynne Robinson}

For the last two summers, university

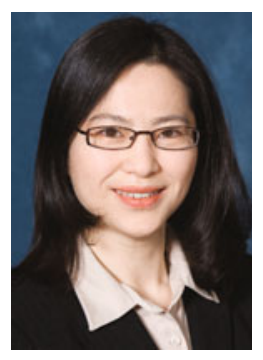

educators have gathered in Ann Arbor, Michigan, for what has been described as a "crash course" on how to incorporate integrated computational Katsuyo Thornton materials engi-
neering (ICME) techniques into undergraduate materials science and engineering (MSE) curricula. Katsuyo Thornton, a founding organizer of the University of Michigan's highly successful Summer School for Integrated Computational Materials Education, is now taking a streamlined version of the program on the road as a short course paired with the $2^{\text {nd }}$ World Congress on ICME (ICME 2013), to be held July 7-11 in Salt Lake City, Utah.

The short course, scheduled for July 11 and 12 at the conclusion of ICME 2013, highlights two of the four modules offered in last year's Summer School, focusing primarily on general concepts and hands-on experience with advanced computational tools. While not the immersive experience of the Summer School, Thornton believes that the short course will serve as a valuable introduction for individuals who are just beginning their own exploration of the potential of ICME in academia, national laboratories or industry. Specific topics covered include computational thermodynamics and density-functional-theory, as well as brief overviews of computational kinetics and computational mechanics.

The impetus for the Summer School was a survey of chairs and department heads of university materials programs, faculty members engaged in computa- tional research, and employers of materials scientists and engineers. "We found that there were challenges that MSE departments faced in evolving the undergraduate curriculum. This included limited resources and faculty availability, as well as limits on the number of courses that could be included in undergraduate degree requirements," Thornton said. "We also found that there were free resources that were being underutilized. The Summer School was established to ease implementation of computational modules into existing standard MSE courses by providing materials and training for overcoming these barriers."

Mark R. DeGuire, Associate Professor, Case Western Reserve University, found the June 2012 Summer School experience particularly helpful when he was tasked to chair a committee responsible for implementing a major revision in the MSE undergraduate curriculum. "Going in, I had only passing familiarity with ICME, and only in a few areas where it is used primarily for research," he said. "The Summer School informed me enough about ICME to help faculty make more informed decisions about how we could implement ICME in the curriculum. It showed me how to use platforms that provide access to public domain ICME tools and also gave us a wealth of curricular modules that could form parts of our planned ICME coursework."

Alessandro Mottura, Birmingham Fellow and Lecturer, University of Birmingham, echoed the value of what he learned as a postdoctoral researcher when he attended the 2011 Summer School. "Getting a bird's eye view of a variety of modeling methods really enriched my research perspective," he said. "It took me to the point of being confident enough to organize a comprehensive materials modeling course."

Mottura plans to use what he learned to increase the ICME content in his university's curriculum. "I am certain this will benefit the students as well as their future employers," he said.

Thornton said that feedback from Summer School students such as DeGuire and Mottura has factored into the ongoing refinement of the curriculum. "We evaluate participants' opinions on how easy it is to implement the modules and what educational values they offer," she said. "The former is essential since our goal would not be met if the modules are not utilized, and the latter is important since these modules are aimed at developing more in-depth understanding of key materials science concepts."

For those who participate in the short course and are interested in learning more, Thornton noted that the full Summer School will travel next to the University of California, Berkeley, in 2014 before returning to Michigan in 2015. Offering the program in varying lengths and at different locations is part of an overall strategy for encouraging broader participation of educators and employers in the basics of ICME teaching and training. Thornton looks to the day, however, when such an effort might not be necessary.

"I hope one day that all MSE curricula will incorporate a substantial computational component and all future materials scientists are familiar with a standard set of computational materials tools," she said. "I believe that the Summer School plays a major role in achieving this goal."

For additional information on this short course, visit the ICME 2013 website at www.tms.org/icme2013. 